\title{
Temporal analysis for implicit compensation of local variations of emission coefficient applied for laser induced crack checking
}

\author{
by G. Traxler*, P. Thanner*, G. Mahler** \\ * PROFACTOR GmbH, Machine Vision, A-1150 Vienna, Austria, gerhard.traxler@profactor.at \\ ** InfraTec GmbH, D-01217 Dresden, Germany, g.mahler@infratec.de
}

\begin{abstract}
This paper presents a new method using implicit compensation of local emission coefficients variations for thermographic inspection. Especially for inspection of metallic sample parts, which commonly have low emission coefficients, temporal signal analysis allows the compensation of disturbing influences caused by emission and reflection. An amplitude independent evaluation method of temporal analysis will be described. Results achieved by using different excitation methods such as laser spot and laser line are presented and compared with results from standard evaluation methods such as gradient evaluation. Additional, experimental setup will be depicted together with results from tests with several metallic sample parts.
\end{abstract}

\section{Introduction}

Laser induced heat flux thermography is a well-known method used for automatic crack checking solutions. In case of the inspection of metal parts thermographic systems have to deal with low and locally different emission coefficient values. These variations of the emission coefficient cause signal distortions which challenge automatic evaluation methods, especially when the parts to be inspected are of a complex geometry and vary in surface treatment.

State of the art evaluation methods which are used for crack detection are often based on local gradient values $\left[{ }^{1}\right]$ with hot spot identification. The gradient method calculates pixelwise local orthogonal gradients, using for example a $3 \times 3$ filter kernel and transposes the results into amplitude and direction values which can be represented as images. This method suffers from the problem that signals and distortions will be amplified. This means that in many cases these methods provide no or insufficient information for reliable discrimination between signals caused by cracks and pseudo signals generated by emission coefficient variations.

This new approach evaluates not only the spatial information but also the temporal behavior of thermal signals. Due to its intrinsic noise suppression the proposed temporal analysis method is expected to provide more accurate crack detection results. Results calculated from both methods gradient and temporal evaluation will be compared and their robustness will be determined.

\section{Temporal Analysis}

The temporal analysis method focuses on the evaluation of temperature over time. The algorithm analyses the whole sequence of thermal images pixel by pixel in order to get the temporal temperature development for each single pixel. Within each of these temporal temperature profiles the moment of maximum amplitude is identified. The method of measuring time of maximum temperature at a distant position from the heating zone is known in context of thermal diffusivity measurement $\left[{ }^{2}\right]$, but will be used here to compensate the disturbing influence of local emissivity.

Starting with the begin of laser heating, heat flows into the surrounding material. Depending on the distance between laser spot center position and the point to be considered the maximum temperature is reached at different moments. In a simple case of a point source, the temperature distribution is given by a so called fundamental solution of the thermal diffusion equation[3]:

$$
\Delta \mathrm{T}_{(\mathrm{r}, \mathrm{t})}=\frac{\mathrm{Q}}{\rho \mathrm{c}_{\mathrm{P}}} \frac{2}{(4 \pi \kappa \mathrm{t})^{3 / 2}} \mathrm{e}^{-\frac{\mathrm{x}^{2}}{4 \kappa \mathrm{t}}}
$$

With $\mathrm{x}[\mathrm{m}]$ as distance from the point source, $\mathrm{t}[\mathrm{s}]$ as time, starting at 0 s with the source puls, $\mathrm{Q}[\mathrm{J}]$ as source energy, $\rho$ $\left[\mathrm{kg} / \mathrm{m}^{3}\right]$ as density, $\mathrm{c}_{\mathrm{p}}[\mathrm{J} / \mathrm{kg} / \mathrm{K}]$ as the specific heat capacity and $\kappa\left[\mathrm{m}^{2} / \mathrm{s}\right]$ as thermal diffusivity the temperature field is defined. In the real application the laser is switched on for a short finite duration, but the characteristic at locations distant to the laser is the same as described in (1). However, care must be taken, if laser-on duration gets into the order of signal maximum time, or if the observed distance gets into the order of the spot size. Fig. 1 shows typical temporal signal evolution at distances of 2.5, 3.0, 4.0 and $7.0 \mathrm{~mm}$ from the position of laser excitation. In this example the signal 
maxima are reached between 257 and 2020ms after excitation. These signals show two effects. First, the bigger the distances the longer it takes until the maximum value is reached. And second, the bigger the distances the smaller the signal amplitudes are.

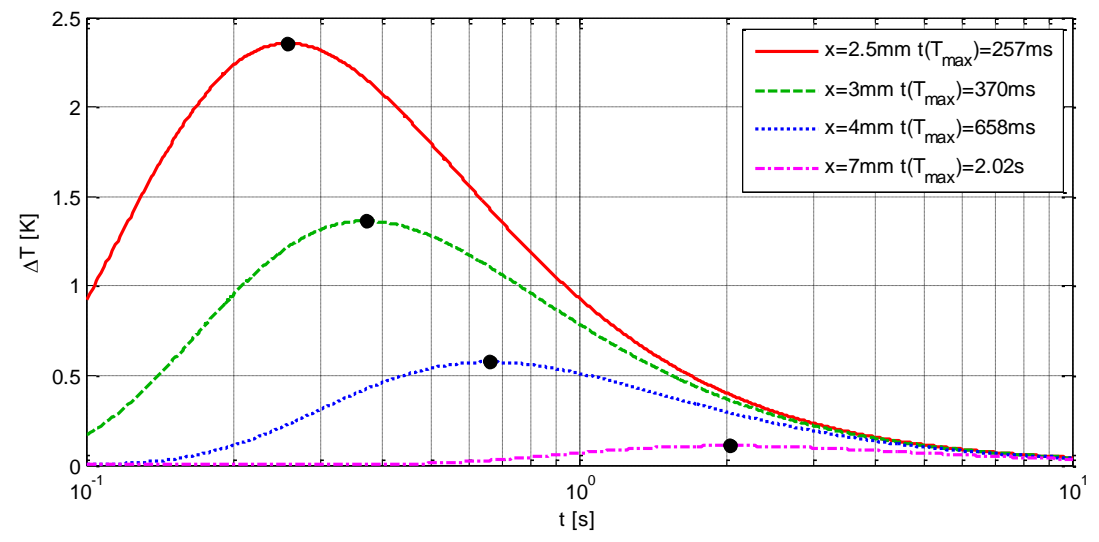

Fig. 1. Temporal evolution at different distances to center point source

A crack causes changes within the heat flux. In metal parts a crack acts as a heat barrier which leads to delayed temperature evolution behind the crack. Using the temporal analysis method each pixel is represented by its time at which signal maximum is reached. Using a linear fit with a proper smoothing function increases the robustness. This is especially important for more distant and therefore low amplitude points. Based on this data cracks can be identified.

Emission coefficient variation as well as reflections from the environment interferes with the amplitude of the thermal signal. The emission coefficient acts as a multiplicative factor which we assume to be constant for each pixel during the time period of thermogram sequence. Although this statement may not be true under all circumstances, but in case of metals, which is the most important application for crack detection, within a temperature range from $20^{\circ} \mathrm{C}$ to $100^{\circ} \mathrm{C}$ the error is insignificant. Reflections from ambient radiation act as additive offset to the thermal signal. As long as ambient conditions are constant for capturing period these disturbances are eliminated when using temporal analysis.

\section{Setup}

The hardware components consist of a $100 \mathrm{~W} \mathrm{CO}$ laser source, a high resolution thermal camera, a processing unit, a robot for positioning the test part to the inspection position and the test part itself. The specific parameters of the laser depend on the thermodynamic properties of the test sample. In this specific case the laser works with a spot diameter of $0.3 \mathrm{~mm}$ and pulse duration of some $1 / 10$ seconds. Once the testing sample has been positioned, the laser is switched on and the thermal camera captures $300 . .500$ images with a frame rate of $200 \mathrm{~Hz}$. The thermal sequence was evaluated offline by a Matlab ${ }^{\circledR}$ program.

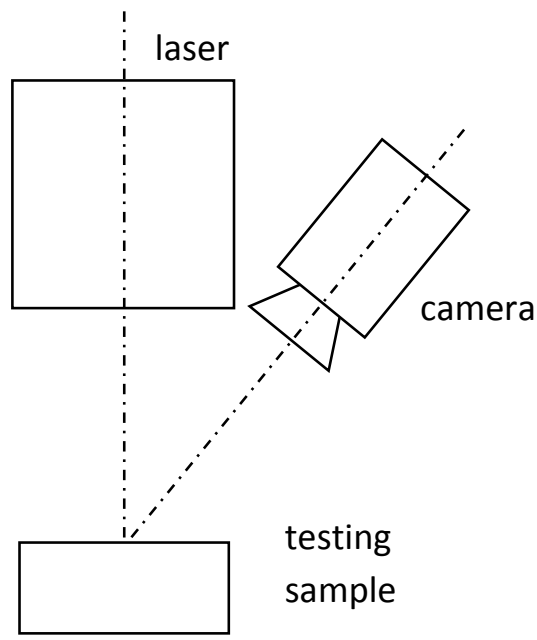

Fig. 2. Composition of main components

\footnotetext{
Matlab is a registered trademark of Mathworks
} 
The laser includes a deflection unit, which controls the position of the laser spot. The spot can be moved with up to $10 \mathrm{~m} / \mathrm{s}$ to form a heating line. In case of short lines with length of approximately $20 \mathrm{~mm}$, as used in the experiments, it takes the spot a little more than $2 \mathrm{~ms}$ to move from one end of the heating line to the other. Due to a short integration time $t=1 . .2 \mathrm{~ms}$ of the camera, the spot will be integrated to $\mathrm{a}$, more or less, solid line. This is also true for the temperature profile in the testing sample, because heat induced by the laser spot won't be distributed over more than $0.3 \mathrm{~mm}$ in one movement cycle of the laser. The laser power is the same in case of a spot, but in case of a line it is distributed along the line, and the radiant incidence is smaller. Therefore the temperature raise is smaller in case of a line. For the experiment the laser power and the spot size were adjusted to avoid temperatures above $100^{\circ} \mathrm{C}$ at the surface of the testing sample.

\section{Experiment with a laser line}

As a first example a laser-line, approximately $1 \mathrm{~mm}$ away from an artificial crack is used to heat up a testing steel sample having a medium emission coefficient of more than 0.6 and produced by casting. The shape of the laser spot-profile is close to a Gaussian distribution with a standard deviation of $0.3 \mathrm{~mm}$. Due to the periodical movement of the spot along a line with high frequency, done by a system of mirrors, the thermal image integrates the spot to a line, as shown in the left thermogram in Fig. 3.
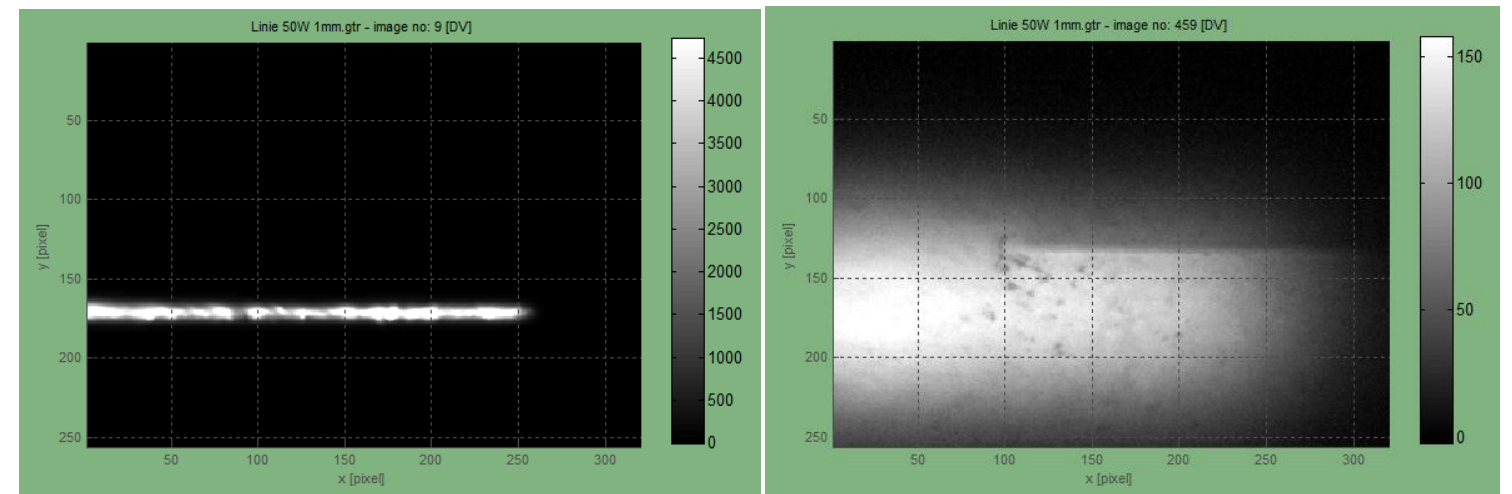

Fig. 3. Thermogram, taken immediately after switching the laser on (left) and after heat has distributed

In the right thermogram in Fig. 3, taken after switching off the laser, heat has already distributed some $\mathrm{mm}$ away from the heating line. The artificial crack is a saw-cut with a depth of $1 \mathrm{~mm}$, as usually used in eddy current systems for testing and verification. It is orientated horizontally at image location $y=130$. Caused by the limited depth of the sawcut, heat flew not only around the crack, but also below it. According to the theory of heat conduction in solids a smooth distribution would be expected in the left thermogram, which is true in a macroscopic scale, but a closer look at the image shows some dark surface patches. They are not an effect of locally colder surface temperature, but an effect of a locally smaller emission coefficient. It suggests itself to calculate the absolute value of the local gradient $g_{(x, y)}(2)$ for evaluation of the crack.

$$
\left|g_{(x, y)}\right|=\sqrt{\left(\frac{d D L_{(x, y)}}{d x}\right)^{2}+\left(\frac{d D L_{(x, y)}}{d y}\right)^{2}}
$$

$D L_{(x, y)}$ is the digital level of the pixel at location $(x, y)$, with $\mathrm{x}$ and $\mathrm{y}$ in units of pixel-position. The result of $(2)$ is shown in Fig. 4. 


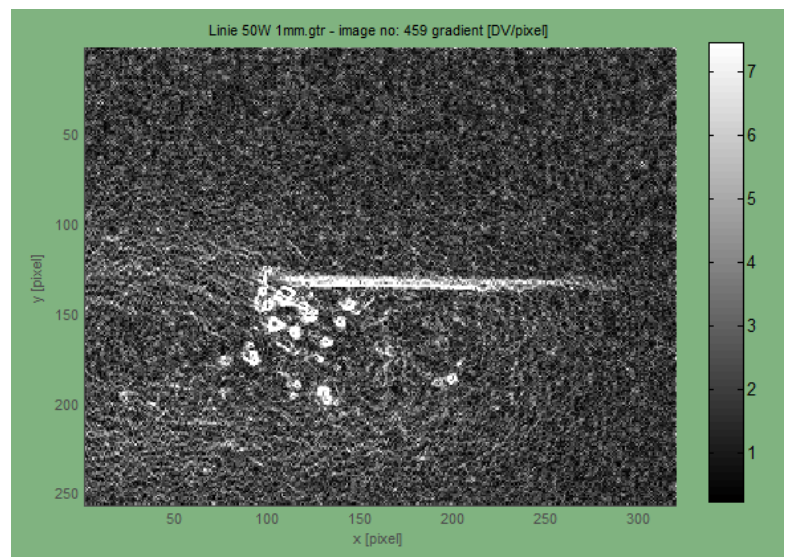

Fig. 4. Gradient-image of thermogram

It is remarkable, that gradient amplitude has almost the same value for the crack and the local distortions caused by the emissivity. In automated evaluation, for instance by threshold and segmentation positive false results will be the consequence. In this special case some filtering could improve robustness of evaluation, but assuming that we plan to detect shorter cracks too, filtering wouldn't help anymore. Evaluation of the entire sequence for the duration of cooling down neither would help. One could take the mean value of the single gradient image sequence to reduce noise, but the effect of local emissivity wouldn't vanish, because of its non-statistical characteristic.

As stated in chapter "Temporal analysis", the influence of local emissivity should be neutralized, if time of maximum signal amplitude is calculated. Fig. 5 is showing two examples for signal evolution, one at a location 20 pixel away from the laser line (left diagram), and the other for a location 75 pixel away from laser line.
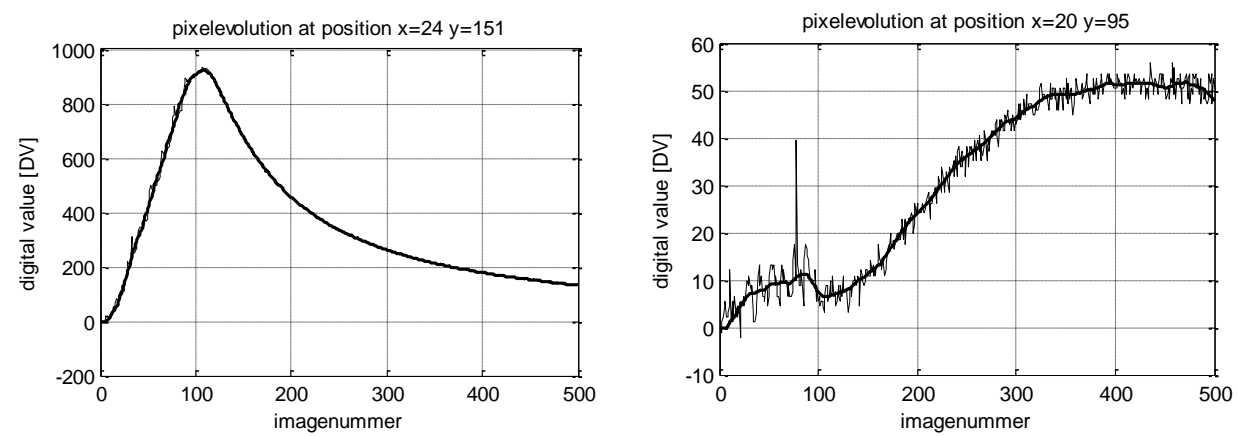

Fig. 5. Signal evolution at closer (left) and more distant (right) location

For improvement of stability some filters were applied to the signal. A median with a length of 15 to eliminate outliers, and additionally smoothing was done by averaging. The original signal in Fig. 5 is represented by the thin line, and the filtered one by the thick line. Next, the time of the global maximum amplitude is calculated. Applying this procedure to each pixel results in an image of maximum time (Fig. 6, left image), or like done here in an image of image numbers, which is just another unit for time.
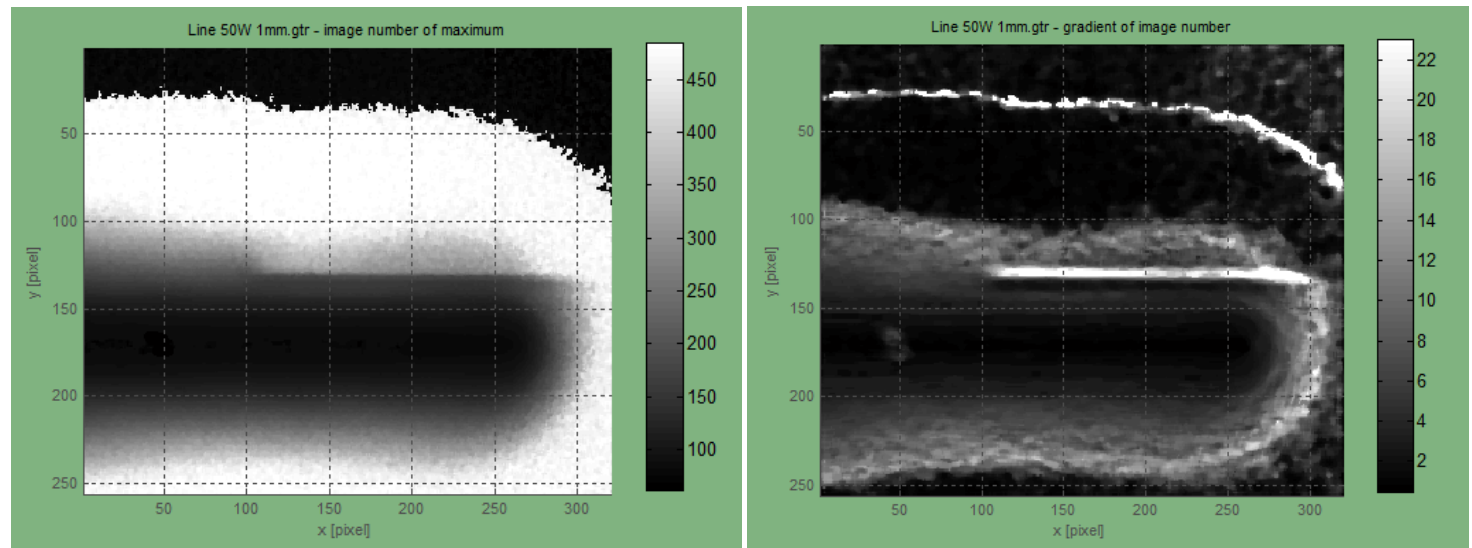

Fig. 6. Image of image numbers for local maximum temperature (left) and gradient image (right) 
For discussion and comparison the temporal image is converted into a gradient image (Fig. 6, right image). The crack at location $\mathrm{y}=130$ is of high amplitude and continuous. Compared to the result of the pure gradient method, in which the crack seems to vanish at the right side, it stays strong after temporal evaluation. As expected no artifacts from local emissivity occur in temporal evolution, but other abnormalities, like a kind of outline-region around the laser line and an additional bright line at $y=30$, appear. The bright line is simply the limit, at which no maximum time can be calculated anymore, because the location is too far away from the laser to find temperature differences. Even below that limit, in the region $y=30 . .80$ the image numbers are saturated at the largest number of the thermal sequence. From evaluations point of view it means, that temperature didn't reach its maximum inside of acquisition time. Therefore it is a restriction for the size of the area around the laser which can be evaluated. Please note, that from this information the dimension of checked area might be calculated. If projected onto the surface of the testing sample it can be used to verify, if the entire surface has been checked without voids. Further it could be sent to the controlling unit of the checking machine to calculate the next checking location preventing some gaps. Both aspects are important for automated checking, and not contained in the non-temporal method. On the other hand the brighter region around the laser impedes evaluation of cracks. We therefore propose to apply some distance dependent weighting, in order to give values farer away from the laser less importance.

\section{Experiment with a laser spot}

Another experiment was done with a semiconductor laser spot of $50 \mathrm{~W}$, a profiles standard deviation of $0.5 \mathrm{~mm}$ and with a wavelength of $810 \mathrm{~nm}$. A thermal sequence of 300 images of a cast iron part with a natural crack was taken for evaluation.
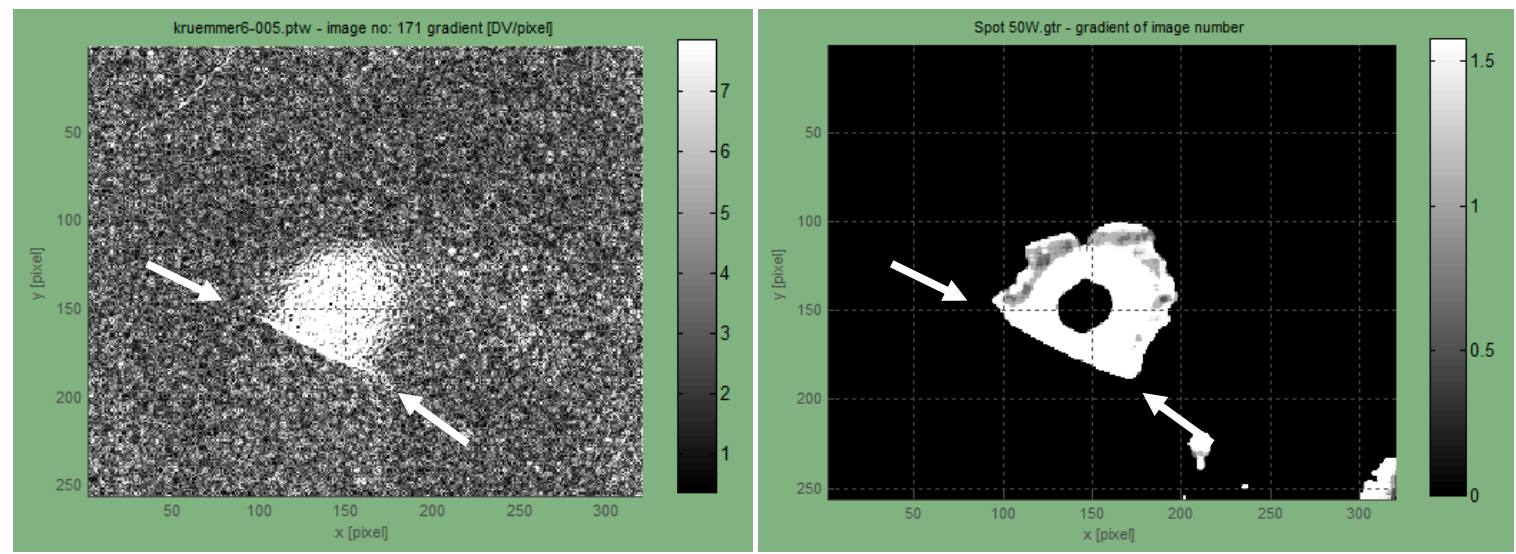

Fig. 7. Gradient method (left) and gradient after temporal evaluation (right)

Fig. 7 shows the results of gradient- and temporal method. Both do not show satisfying quality for automated evaluation, even if the crack is recognizable by the viewer (between arrows). The reason for the low quality is a highly reflecting surface of the testing sample, and in consequence a low temperature raise in the laser spot. In case of temporal evaluation the sequence is too short. The crack is located at the position of the outline of the evaluable area, and there is no temperature raise outside this area. To solve this problem a longer sequence would have to be acquired, but this example, which of course was done with worse parameters, is pointing out an important restriction, when applying the temporal method.

\section{Experiment with complex geometry}

Next experimental tests were performed on a highly reflecting steel tube with periodically smoothly alternating diameters in the range from 28 to $34 \mathrm{~mm}$ as sample part. The crack within the tube is straight, has a length of approximately $1.5 \mathrm{~mm}$ and is a natural one, generated in a welding process. The thermogram sequence has been captured at $50 \mathrm{~Hz}$, and a laser spot with $0.3 \mathrm{~mm}$ of standard deviation was deflected by a mirror system to form a straight line. 


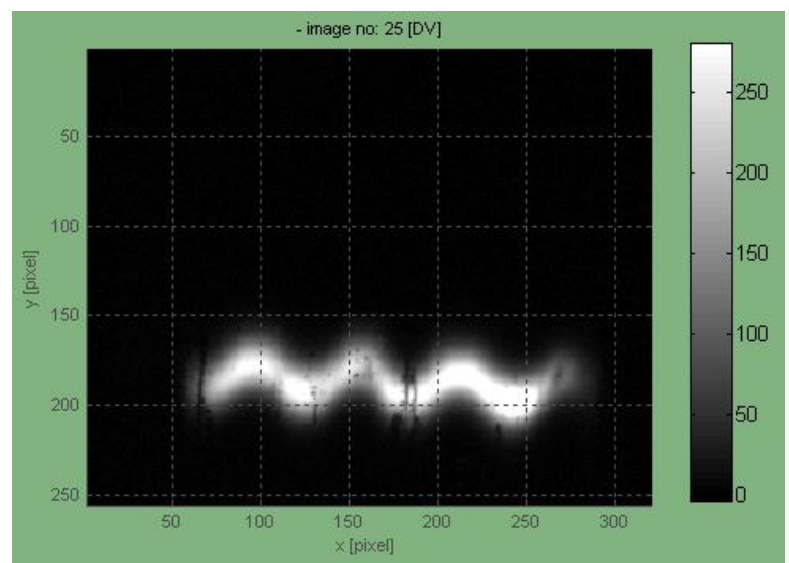

Fig. 8. Temperature distribution immediately after laser was switched off

Even if a straight laser line was projected onto the surface of the testing sample it looks like a sine-wave in the thermogram (Fig. 8). It is caused by the 3-dimensional surface and the angle between laser and camera, which is approximately $45^{\circ}$. This effect is very familiar to a laser-triangulation for distance measurement, and in turn, the composition of laser, camera and testing sample is exactly the same.
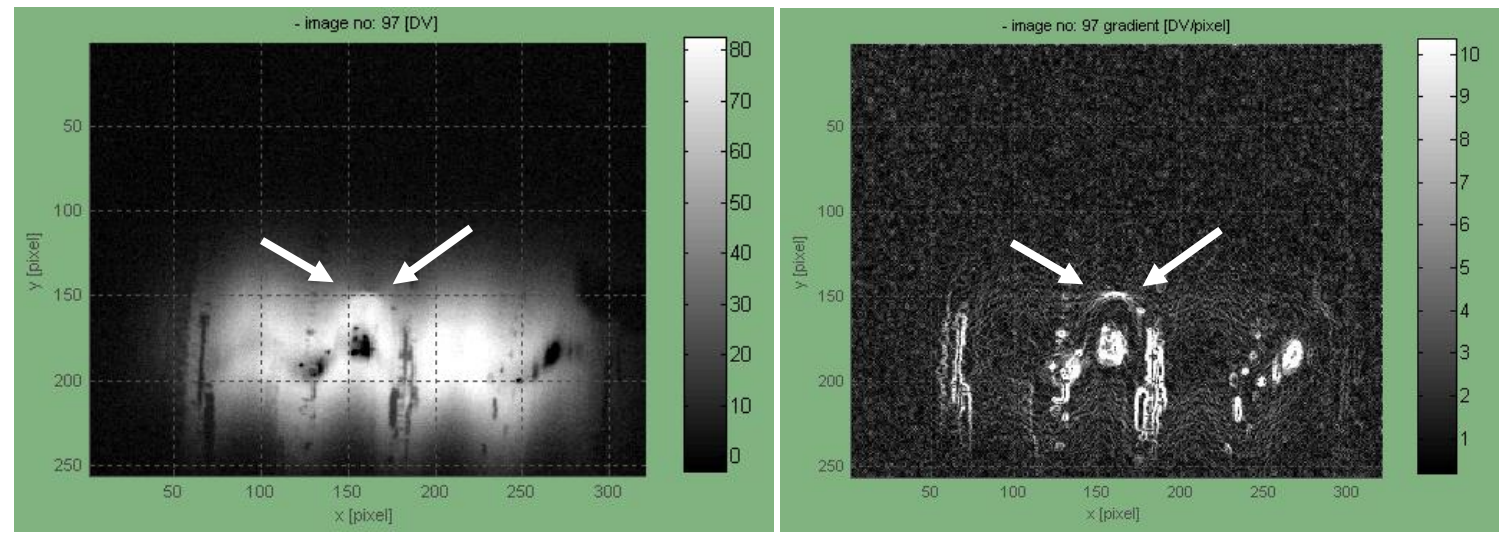

Fig. 9. Thermogram after heat has distributed (left) and gradient (right)

In the thermogram in Fig. 9 a lot of small areas with low emissivity can be identified by local dark spots, and a lot of abnormalities in the gradient image are the consequence. In this case filtering isn't able to solve the problem, because of the crack, marked by the arrows being small in relation to the errors caused by locally low emissivity. Some of the abnormalities are not caused by local emissivity, but by the surface structure at locations where it is not flat or rather at locations, which look like edges in the cameras view. The amplitude of this edge-signal is smaller than the one caused by emissivity, but higher amplitudes can be assumed in case of sharper edges.

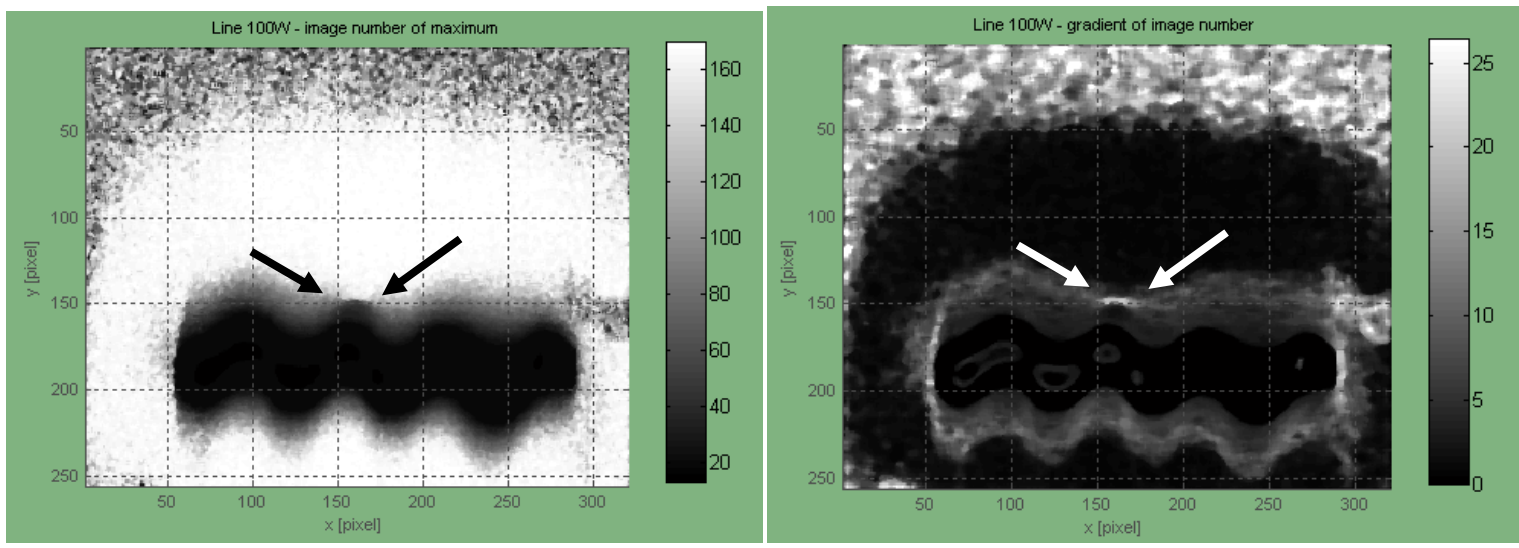

Fig. 10. Temporal evaluation (left) and gradient of temporal evaluation (right) 
In the temporal evaluation in Fig. 10 neither effects of low emissivity spots, nor effects of edges can be recognized. The crack is not distorted by the perspectival distortion. It has high amplitude and a sharp outline. Disadvantageously two lines at the location of the right and left end of the laser line could complicate identification of cracks. This effect has to be solved by masking out the two regions.

\section{Conclusions}

The effect of locally varying emissivity has successfully been suppressed by temporal method. Further the checkable area can be derived from data, already existing from evaluation, even if there is no crack present. Some restrictions could be identified as limitation of the method as they are:

- $\quad$ Observation time must be long enough to avoid additional clipping of the evaluable area.

- $\quad$ Artifacts occur at the ends of the laser line.

- In regions of lower amplitude, there is some remainder in the gradient image, which could complicate identification of cracks.

- $\quad$ For a robust identification, weighting of evaluated data in order to enhance data near the laser spot and to decrease data farer away from it, seems to be necessary.

Omitting the advantage of getting the checkable area, results of gradient and temporal method delivers comparable results for laser induced crack checking, if the surface of the testing sample has a high and homogeneous emissivity. Otherwise the temporal method is proposed to avoid problems caused by inhomogeneous emissivity.

\section{Outlook}

Some improvement could be possible by comparison of profiles from temporal evaluation with a fitted polynomial of second order, since profiles are expected to have the characteristic of a parabola.

\section{Acknowledgment}

This work was supported by the European Commission in the 7th Framework program under grant No. 284607, project ThermoBot.

\section{References}

\footnotetext{
${ }^{1}$ B.O.Tranta, Thermoinductive investigations of magnetic materials for surface cracks, Quantitative InfraRed Thermography Journal, Vol.1 Issue 1, 2004

${ }^{2}$ Infrared Methodology and Technology, 1994, ISBN 2-88124-590-0, p153, V.P.Vavilov

${ }^{3}$ H.S.Carslaw, J.C.Jaeger, Conduction of Heat in Solids, 2nd Edition 1959, Oxford University Press, ISBN 0-19$853368-3, \mathrm{p} 256$
} 